\title{
Systematic screening and identification of novel psoriasis-specific genes from the transcriptome of psoriasis-like keratinocytes
}

\author{
ZHEN WANG $^{1 *}$, HUAPING ZHENG $^{1 *}$, HONG ZHOU $^{1 *}$, NONGYU HUANG $^{1}$, XIAOQIONG WEI $^{1}$, \\ XIAO LIU ${ }^{1}$, XIU TENG ${ }^{1}$, ZHONGLAN HU ${ }^{1}$, JUN ZHANG ${ }^{1}$, XIKUN ZHOU ${ }^{1}$, WEI LI $^{2}$ and JIONG LI ${ }^{1}$ \\ ${ }^{1}$ Department of Biotherapy and Cancer Center; ${ }^{2}$ Department of Dermatovenereology, West China Hospital, Sichuan University, \\ and Collaborative Innovation Center for Biotherapy, Chengdu, Sichuan 610041, P.R. China
}

Received March 28, 2018; Accepted November 5, 2018

DOI: $10.3892 / \mathrm{mmr} .2018 .9782$

\begin{abstract}
Psoriasis is a chronic inflammatory skin disease. Keratinocytes (KCs), as skin-specific cells, serve an important role in the immunopathogenesis of psoriasis. In the present study, transcriptome data derived from psoriasis-like $\mathrm{KCs}$ were used together with the reported transcriptome data from the skin/epidermis of patient with psoriasis, excluding known psoriasis-associated genes that have been well described in the previous studies according to GeneCards database, to screen for novel psoriasis-associated genes. According to the human expressed sequence tag of UniGene dataset, six genes that are located near psoriasis-associated loci were highly expressed in skin. Among these six genes, four genes (epiregulin, NIPA like domain containing 4 , serpin family B member 7 and WAP four-disulfide core domain 12) were highly expressed in normal mouse epidermis (mainly $\mathrm{KCs}$ ) and mouse psoriatic epidermis cells, but not in psoriatic dermis cells, which further emphasized the specificity of these genes. Furthermore, in systemic inflammatory response syndrome (SIRS), SERPINB7 showed no difference in expression in immune-activated tissues from SIRS and control mice. It was also found that the mRNA expression levels of SERPINB in lesional skin of patients with psoriasis were significantly higher than in non-lesional psoriatic skin from the same patients. SERPINB7 may be a valuable candidate for further studies. In the present study, a method for identifying novel key pathogenic skin-specific molecules is presented, which may be used for investigating and treating psoriasis.
\end{abstract}

Correspondence to: Professor Jiong Li, Department of Biotherapy and Cancer Center, West China Hospital, Sichuan University, and Collaborative Innovation Center for Biotherapy, 1 Keyuan 4th Road, Gaopeng Street, High Technological Development Zone, Chengdu, Sichuan 610041, P.R. China

E-mail: lijionghh@scu.edu.cn

${ }^{*}$ Contributed equally

Key words: systematic screening, psoriasis, skin-specific genes, keratinocytes, local immunity

\section{Introduction}

Skin functions as an important natural barrier between an organism and its external environment, thus it has a unique biological structure and specific immune functions (1). Skin is composed of two distinct regions, the epidermis and dermis. The predominant cell type of the epidermis is keratinocytes (KCs), whereas the dermis contains cells of the immune system, including dendritic cells (DCs), T helper cells, $\gamma \delta$ T cells, natural killer T cells, macrophages and fibroblasts (2). A skin immune system imbalance can cause several immune-mediated skin diseases, such as psoriasis (1). Therefore, in-depth analysis of the skin immune system would be of great clinical significance for the treatment of these diseases.

Psoriasis is a common chronic inflammatory skin disease triggered by a dysregulated immune response, which typically manifests as plaques with adherent silvery scales and has a great impact on the physical and mental health of patients $(3,4)$. This disease is characterized by excessive growth and aberrant differentiation of KCs. In psoriasis, the pathological changes in KCs include hyperkeratosis, parakeratosis, a loss of the normal granular layer and acanthosis with elongation of the epidermal rete ridges (5). Thus, as the skin specific cells, $\mathrm{KCs}$ are the main cell type affected by psoriasis.

The immunopathogenesis of psoriasis outlines the important role KCs serve in the induction and amplification of psoriatic inflammation. KCs respond to different signals and release the cathelicidin antimicrobial peptide (LL-37), which activates plasmacytoid DCs (pDCs) via Toll-like receptors (5-7). Myeloid DCs are activated by interferon (IFN)- $\alpha / \beta$ from the $\mathrm{pDCs}$, stimulating psoriatic $\mathrm{T}$ cells that produce interleukin (IL)-17, IFN- $\gamma$ and IL-22. KCs also produce chemokines and antimicrobial peptides, which attract myeloid DCs and T cells that produce IL-17. The cytokines released by these cells further stimulate the $\mathrm{KCs}$, and the immune circuit is further amplified by feedback cytokines produced by the KCs $(1,5,8)$. Thus, KCs have a central role in the immune circuits of psoriatic skin.

Several immunosuppressants are currently available for psoriasis treatment; their targets include cytokines, signaling molecules and receptors in the immune system (7). However, as with any drug designed to suppress the general immune system, the main concern is the risk of serious side effects. 
Efalizumab, a monoclonal antibody targeting cluster of differentiation (CD)11a, has inhibitory effects against broad $\mathrm{T}$ cell subsets, resulting in systemic immune suppression, which can lead to serious infection, cancer and other severe complications $(9,10)$. As a result, it was withdrawn 6 years after approval (FDA Issues Boxed Warning for Efalizumab, 2008) (11). The clinical development of briakinumab, an antibody targeting IL-23/P40, was halted after a series of major cardiovascular events occurred during clinical trials (9). In clinical application, other psoriasis drugs, including infliximab, [a tumor necrosis factor (TNF)- $\alpha$ blocker], corticosteroids, antibiotics and vitamins, can also cause different degrees of toxicity (12). In addition, IL-17-targeting antibodies and small molecule drugs targeting Janus kinases also have limitations in the treatment of psoriasis. The most fundamental reason is that these drugs do not have specific targets or cannot regulate the cutaneous immunity $(13,14)$. Therefore, identifying novel psoriasis-specific molecules will help treatment.

In the present study, novel psoriatic skin-specific genes were screened from the transcriptome of psoriasis-like KCs. An integrative approach, combining psoriatic transcriptome data, psoriasis-associated genes information, genetic loci linked to psoriasis and human tissue expression pattern, was used to screen novel psoriasis-associated genes that were highly expressed in the skin/epidermis. The present study provides a novel way of identifying novel skin-specific genes for cutaneous diseases.

\section{Materials and methods}

Human skin samples. The present study was performed in accordance with the principles of the Helsinki Declaration and approved by the Ethics Committee of the West China Hospital, Sichuan University (Chengdu, Sichuan, China). Written informed consent was obtained from all study participants prior to the study. All patients were diagnosed based on the clinically apparent symptoms (fairly easily diagnosed as characteristic red colored plaques with well-defined borders and silvery-white dry scale), and histopathological criteria (abnormal proliferation and differentiation of the epidermis, hyperkeratosis and parakeratosis of keratinocytes) (5). All patients were assessed according to the Psoriasis Area and Severity Index (15). Skin samples $\sim 0.5-1.0 \mathrm{~cm}$ were collected from eight patients with psoriasis (four females and four males; age, 21-63 years) at the West China Hospital, Sichuan University between March 2016 and November 2017. The lesional and non-lesional psoriatic skin $(\sim 0.5-1.0 \mathrm{~cm})$ were taken from each patient, one was obtained from lesional skin of patients and the other from non-lesional skin of the same patients. The fresh skin samples were snap-frozen in liquid nitrogen and stored at $-80^{\circ} \mathrm{C}$. All participants had not been treated with systemic therapy including investigational agents for at least 4 weeks prior to the study entry. Patients with a history of other autoimmune diseases, immunologic deficiency diseases or tumors were excluded.

Cell culture. The human HaCaT KCs were obtained from the China Center for Type Culture Collection (Wuhan, China; CCTCC no. 0106). HaCaT cells were cultured in Dulbecco's modified Eagle's medium (DMEM; Gibco; Thermo Fisher
Scientific, Inc., Waltham, MA, USA) supplemented with $10 \%$ (v/v) fetal bovine serum (FBS; Gibco; Thermo Fisher Scientific, Inc., Waltham, MA, USA), $100 \mathrm{U} / \mathrm{ml}$ penicillin G and $0.1 \mathrm{mg} / \mathrm{ml}$ streptomycin sulfate (Thermo Fisher Scientific, Inc.). The conditions of cell culture were $37^{\circ} \mathrm{C}$ and $5 \% \mathrm{CO}_{2}$. All cells were demonstrated to be free from mycoplasma contamination.

Induction of psoriatic keratinocytes model in vitro. The five proinflammatory cytokines (termed M5), including IL-17A, oncostatin-M, TNF- $\alpha$, IL-22, and IL-1a (ProSpec Bio, East Brunswick, NJ, USA) were used to induce psoriasis-like KCs inflammation that recapitulates the features of psoriasis (16), as described in a previous study (17). Briefly, KCs were cultured to $80 \%$ confluency and then starved for $24 \mathrm{~h}$ in DMEM without serum prior to stimulation. The cells were then stimulated with $10 \mathrm{ng} / \mathrm{ml}$ recombinant IL-17A, $10 \mathrm{ng} / \mathrm{ml}$ recombinant oncostatin-M, $10 \mathrm{ng} / \mathrm{ml}$ recombinant TNF- $\alpha, 10 \mathrm{ng} / \mathrm{ml}$ recombinant IL-22 and $10 \mathrm{ng} / \mathrm{ml}$ recombinant IL- $1 \alpha$ in combination or untreated for $24 \mathrm{~h}$ prior to the whole transcriptome gene expression analysis.

Microarray expression profiling. Gene array analysis was performed using Human Expr 12x135K Arr Del (Roche-NimbleGen; Roche Diagnostics, Basel, Switzerland) by KangChen Bio-tech, Inc. (Shanghai, China). In brief, total mRNA was isolated from $\mathrm{KCs}$ at $24 \mathrm{~h}$ post-stimulation with M5 using PureYield ${ }^{\text {TM }}$ RNA Midiprep System (Promega Corporation, Madison, WI, USA). Total RNA was quantified by the NanoDrop ND-1000A, and RNA integrity and gDNA contamination was assessed by standard denaturing $1 \%$ agarose gel electrophoresis. Total mRNA of each sample was used for labeling and array hybridization were performed according to the manufacturers' protocols: Reverse transcription using SuperScript Double-Stranded cDNA Synthesis kit (Thermo Fisher Scientific, Inc.); ds-cDNA labeling with NimbleGen one-color DNA labeling kit (Roche Diagnostics, Mannheim, Germany); array hybridization using the NimbleGen Hybridization System followed by washing with the NimbleGen wash buffer kit (Roche Diagnostics); array scanning using the Axon GenePix 4000B microarray scanner (Molecular Devices LLC, Sunnyvale, CA, USA). Raw signal intensities were extracted and normalized using the Robust Multichip Average (RMA) method by NimbleScan v2.5 software (Roche NimbleGen Inc., Madison, WI), and low intensity $(<100.0)$ genes were filtered. Further data analysis was performed using Agilent GeneSpring GX 11.5.1 software (Agilent Technologies, Inc., Santa Clara, CA, USA). Two biological replicates were used for each sample, expression values were normalized based on the mean expression value for each probe set, differently expressed probe sets were identified based on Student's t-test for paired samples' normalized expression values using the following cutoff: Absolute fold change $(\mathrm{FC})>3$ and a $\mathrm{P}<0.01$, false discovery rate $<0.05$. In addition, 3,577 differentially expressed genes (DEGs) were obtained from psoriatic lesional and normal skin in the study by Li et al (18), and 1,446 DEGs in psoriatic lesional epidermis compared with non-lesional psoriatic epidermis in the study by Mitsui et al (19). To compare the present keratinocytes microarray data with previously published reports data, these 
two transcriptome data sets and the transcriptome datase in the psoriasis-like KCs model were further analyzed for enriched Gene Ontology (GO) terms using Gorilla (cbl-gorilla. cs.technion.ac.il/). Biological terms that have many genes in common can be grouped into a module of associated terms and genes, with a significance threshold of 0.001 .

Gene expression datasets. GEO DataSets (GSE40263) were obtain of peripheral blood mononuclear cells (PBMCs) of psoriasis $(n=5)$ and healthy controls $(n=5)$ from National Center for Biotechnology Information (NCBI; https://www. ncbi.nlm.nih.gov/geo/query/acc.cgi?acc=GSE40263). The ID REF of EREG, NIPAL4, SERPINB7 and WFDC12 were retrieved in website of Affymetrix, Inc (https://www.affymetrix.com/analysis/netaffx/index.affx). Based on the gene of ID_REF, RMA signal intensity, which is a form of quantile normalization applicable to gene expression (microarray) experiments, was searched in the data table of psoriasis and healthy controls. The PBMCs expression levels of these four genes in the psoriasis and healthy controls were analyzed.

Sources of genetic loci linked to psoriasis. Genetic loci associated with psoriasis were obtained from a knowledgebase of Human Genes and Genetic Disorders [Online Mendelian Inheritance in Man (OMIM); search, 'psoriasis'; entries with 'gene map locus'; retrieve, 'gene map'; www.omim. org/search?index=entry\&search=psoriasis\&filter=gm_exists\% 3 Atrue\&sort=chromosome_number+asc $\% 2 \mathrm{C}+$ chromosome_ sort+asc \&start $=1 \&$ limit $=100 \&$ retrieve=geneMap; accessed February 2018], and previous research reports prior to February 2018; these included 1p36, 1q21.3, 2p16, 5q15, 5q31.1, 5q33.3, 5q33.3, 8p23.2, 9q34.13, 13q12.11, 13q13.3, 14q13.2, 16p11.2, $17 q 11.2,14 q 32.13,18 q 21.2,18 q 21.33,18 q 22.1,19 p 13,19 q 13.41$ and 20q13 (20-29).

Psoriasis-associated genes in GeneCards database. Using the GeneCards database (www.genecards. org/Search/Keyword?queryString=PSORIASIS; accessed February 2018), psoriasis-associated genes were obtained and the approximate degree of correlation was inferred from the score. A score $\geq 1$ was considered to be a psoriasis-associated gene.

Expressed sequence tag (EST) sources. National Center for Biotechnology Information Unigene (www.ncbi.nlm.nih. gov/unigene/) was used to search the indicated homo candidate genes, their EST profiles were entered and the approximate gene expression of various tissues was inferred from the transcripts per million (TPM). Indicated genes with relatively high specific expression in skin tissue were selected by screening the indicated gene EST profiles inferred from TPM value.

Ethics statement. Wild-type C57BL/6 female mice 8-12 weeks old, weight, 17-18 g, n=180, were purchased from Vital River Laboratory Animal Technology Co., Ltd. (Beijing, China). The animals were housed under the following controlled conditions: $12 \mathrm{~h}$ light-dark cycle at a steady temperature of $25 \pm 1^{\circ} \mathrm{C}$, with free access to water and food. The animal protocols were approved by the Committee on the Ethics of Animal Experiments of Sichuan University. The experimental procedures were conducted according to the ethical Guidelines For The Care And Use Of Laboratory Animals of the National Institutes of Health (https:/grants.nih.gov/grants/olaw/guidefor-the-care-and-use-of-laboratory-animals.pdf) and the International Association for the Study of Pain (IASP). Every effort was made to decrease the number of animals used and to reduce animal suffering.

Tissue dissection. Following the sacrifice of the mice, various tissues (large intestine, lung, liver, testis, ovary, brain, spleen, and kidney, small intestine and heart) were collected as previous described (30). The backs of mice were shaved, the skin was wiped with alcohol prior to its removal, then subcutaneous fat was removed and was cut into small pieces convenient for digestion and separation, and the samples $(\sim 0.3 \mathrm{~cm})$ were then incubated in dispase II $(2.5 \mathrm{U} / \mathrm{ml}$; Sigma-Aldrich; Merck $\mathrm{KGaA}$, Darmstadt, Germany) at $4^{\circ} \mathrm{C}$ overnight followed by immersion in DMEM containing 50\% (v/v) FBS to inactivate the dispase II. The epidermis and dermis were then separated at the epidermal-dermal interface under magnification with a dissecting microscope. Only pieces that consisted entirely of epidermis or dermis were used.

Imiquimod (IMQ)-induced psoriasis-like skin inflammation. The psoriasis animal model used in the present study was the IMQ-induced psoriasis-like skin inflammation. The IMQ mouse model of psoriasis-like skin inflammation was induced as previously described (31). Briefly, the day before induction, the backs of the mice were shaved. Subsequently, the backs of the mice were treated with Aldara cream (Sichuan MingXin Pharmaceutical Co., LTD., Sichuan, China) containing $5 \%$ IMQ (55 mg) once daily for 1-6 days.

Lipopolysaccharide (LPS)-induced systemic inflammatory response syndrome (SIRS). SIRS was induced by intraperitoneal injection of dose of $2.5 \mathrm{mg} / \mathrm{kg}$ of LPS from Escherichia coli 0111:B4 (Sigma-Aldrich; Merck KGaA). Groups of animals were sacrificed $3 \mathrm{~h}$ after a single injection of LPS $(n=5)$ or normal saline (control group, $n=5)$, three independent experiments were performed. Various tissues (large intestine, lung, liver, testis, ovary, brain, spleen, and kidney, small intestine, heart and skin) were collected as previous described (30). The systemic expression of the proinflammatory cytokine TNF- $\alpha$ in various tissues (large intestine, lung, liver, testis, ovary, brain, spleen, kidney, small intestine and heart) were induced by LPS and the systemic inflammatory response was activated.

Reverse transcription-quantitative polymerase chain reaction $(R T-q P C R)$. Cells or mouse tissues (large intestine, lung, liver, testis, ovary, brain, spleen, and kidney, small intestine, heart and skin) were obtained and TRIzol (Invitrogen; Thermo Fisher Scientific, Inc.) was used to extract the total RNA, according to the manufacturer's protocol. Gel electrophoresis was performed to detect the integrity of the total RNA extracted. Total RNA $(2 \mu \mathrm{g})$ was reverse transcribed into cDNA, PrimeScript RT reagent kit with gDNA Eraser (Takara Bio, Inc., Otsu, Japan) was used for RT to produce cDNA at $42^{\circ} \mathrm{C}$ for $50 \mathrm{~min}$ and at $85^{\circ} \mathrm{C}$ for $5 \mathrm{~min}$, according to the manufacturer's protocol. cDNA (20 ng) was subjected 
to qPCR analysis with TB Green ${ }^{\mathrm{TM}}$ Premix Ex $\mathrm{Taq}^{\mathrm{TM}}$ II (Tli RNaseH Plus; Takara Bio, Inc.) according to the manufacturer's protocol. PCR was run under the following conditions: An initial denaturation at $95^{\circ} \mathrm{C}$ for $30 \mathrm{sec}, 35$ cycles of $95^{\circ} \mathrm{C}$ for $5 \mathrm{sec}$, annealing and extension at $60^{\circ} \mathrm{C}$ for $30 \mathrm{sec}$, and final extension at $72^{\circ} \mathrm{C}$ for $5 \mathrm{~min}$. $\beta$-actin was used as the internal control. All primers were obtained from Chengdu Qing Ke Zi Xi Biotechnology Co. (Chengdu, China). Human primers included $C-X-C$ motif chemokine ligand 1 (CXCL1 forward, 5'-GCCAGTGCTTGCAGACCCT-3' and reverse, 5'-GGC TATGACTTCGGTTTGGG-3'), CXCL2 (forward, 5'-CAA ACCGAAGTCATAGCCAC-3' and reverse, 5'-TCTGGT CAGTTGGATTTGCC-3'), CXCL8 (forward, 5'-TCTGTC TGGACCCCAAGGAA-3' and reverse, 5'-GCATCTGGC AACCCTACAACA-3'), C-C motif chemokine ligand 20 (CCL20 forward, 5'-TGACTGCTGTCTTGGATACAC AGA-3' and reverse, 5'-TGATAGCATTGATGTCACAGC CT-3'), CCL27 (forward, 5'-AGCACTGCCTGCTGTACT CA-3' and reverse, 5'-TCTTGGTGCTCAAACCACTG-3'), S100 calcium binding protein A7 (S100A7 forward, 5'-CCT TAGTGCCTGTGACAA-3' and reverse, 5'-CTGCTTGTG GTAGTCTGT-3'), S100A8 (forward, 5'-AGTGTCCTCAGT ATATCA-3' and reverse, 5'-CATCTTTATCACCAGAAT G-3'), S100A9 (forward, 5'-CAACACCTTCCACCAATA C-3' and reverse, 5'-TCATTCTTATTCTCCTTCTTGAG-3'), S100A12 (forward, 5'-CAATACTCAGTTCGGAAGG-3' and reverse, 5'-CTTTGATATTCTTGATGGTGTTT-3'), LL-37 (forward, 5'-GATAACAAGAGATTTGCCCTGCTG-3' and reverse, 5'-TTTCTCAGAGCCCAGAAGCCTG-3'), $\beta$-defensin 2 [BD2 (forward, 5'-TTCTCGTTCCTCTTCATA-3' and reverse, 5'-ATATGGCTCCACTCTTAA-3')], serpinfamily $B$ member 7 (SERPINB7 forward, 5'-TTGGTGAAGGTG GCATAA-3' and reverse, 5'-CAGAGCACTTGGGAGATT-3'), $\beta$-actin (forward, 5'-CCACGAAACTACCTTCAACTCC-3' and reverse, 5'-GTGATCTCCTTCTGCATCCTGT-3'). Mouse primer sequences including epiregulin (EREG forward, 5'-ACC GCCTTAGTTCAGATG-3' and reverse, 5'-ATGTCC ACCAGGTAGATG-3'), NIPA like domain containing 4 (NIPAL4 forward, 5'-GCACCCTGTCTGGCTTCGT-3' and reverse, 5'-AGTTTAATGACTGTGGGCTCTGG-3'), phospholipase A2 group IVE (PLA2G4E forward, 5'-GAT GGTGACAGACTCCTT-3' and reverse, 5'-GCAGCAAAG CCTAAAGTTA-3'), SERPINB7 (forward, 5'-AATAAT CAGCCAGGACTTC-3' and reverse, 5'-CACACTCAA TGTAGTTCTTATG-3'), solute carrier family 1 member 6 (SLC1A6 forward, 5'-GGCATCATCATCTGGTATG-3' and reverse, 5'-GGTGACGAGGAAGTAGATA-3'), WAP four-disulfide core domain 12 (WFDC12 forward, 5'-GAC AACAGTGAAGAACAGAT-3' and reverse, 5'-GGAGTCCAA GATCAAGGT-3'), $\beta$-actin (forward, 5'-CCTCTATGCCAA CACAGTGC-3' and reverse, 5'-ACATCTGCTGGAAGGTGG AC-3'). Relative mRNA expression changes were calculated using the $2^{-\triangle \Delta C q}$ method (32).

Statistical analysis. The data are expressed as the mean \pm standard deviation. All statistical analyses were performed using GraphPad Prism 7.0 software (GraphPad Prism Inc., La Jolla, CA, USA). Comparison between two groups was performed by unpaired Student's t-test. Comparison among multiple groups was performed by one-way analysis of variance followed by a Tukey's post-hoc test. $\mathrm{P}<0.05$ was considered to indicate a statistically significant difference.

\section{Results}

Identification of differential expression genes in psoriatic $K C s$. To induce a psoriasis-like KCs model in vitro, $\mathrm{KCs}$ were stimulated with M5 combination (containing IL-1a, IL-17A, IL-22, oncostatin-M and TNF- $\alpha$ ). The mRNA levels of the chemokines (CXCL1, CXCL2, CXCL8, CCL20 and CCL27) and antimicrobial peptides (S100A7, S100A8, S100A9, S100A12, LL-37 and BD2) were significantly increased by M5 stimulation of KCs (Fig. 1A). The result suggested that an in vitro model of psoriasis-like KCs was established (16). Subsequently, using a human gene expression microarray, a transcriptomic profile of the psoriasis-like KCs was generated. Different colors represent gene expression levels. Scatter plots provided a profile of psoriasis-like KC mRNAs that were upregulated, downregulated or unaffected compared with the control (Fig. 1B). These data identified 2,957 DEGs with a FC $>3(\mathrm{P}<0.01)$ as a cutoff, of which 1,735 were upregulated and 1,222 were downregulated (Fig. 1B).

Comparative analysis of DEGs in psoriatic KCs, human psoriatic skin and human psoriatic epidermis. In previous studies, the DEGs between psoriatic lesional and normal skin samples reported by Li et al (18), and DEGs between lesional and non-lesional epidermis samples reported by Mitsui et al (19), were analyzed further. In the present study, these two data sets were analyzed and compared with the transcriptome dataset in the current psoriasis-like KCs model. GO enrichment analysis was performed on these three transcriptome data sets. The result produced a similar distribution pattern of biological processes and similar genes enrichment among the three datasets (Fig. 1C), indicating that the changes in the biological process observed in psoriasis-like KCs were also presents in patients with psoriasis.

The most significantly enriched biological processes among the upregulated genes included 'cellular response to biotic stimulus', 'cellular response to molecule of bacterial stimulus', 'cytokine-mediated signaling pathway', 'defense response', 'epithelial cell differentiation', 'immune effector process', 'immune response', 'immune system process' and 'innate immune response' (Fig. 1C). By contrast, the most significantly enriched biological processes among the downregulated genes included 'biological adhesion', 'cell adhesion' and 'extracellular matrix organization' (Fig. 1C). All these biological processes have been implicated in psoriasis. These results suggested that the psoriasis-like KCs model is an appropriate model for with similar changes observed as in clinical samples.

Screening of key novel psoriasis-associated genes from differential expression genes that are identified in psoriatic keratinocytes. The human gene expression microarray identified 2,957 DEGs in the induced psoriasis-like KCs model in vitro (Fig. 2, step 1). DEGs in the induced psoriasis-like $\mathrm{KCs}$ model ( $n=2,957$, psoriasis-like vs. normal KCs) were integrated with the DEGs identified in previous studies $[n=3,577$, psoriatic lesions vs. normal skin] (18); and $n=1,446$, psoriatic lesional 
A

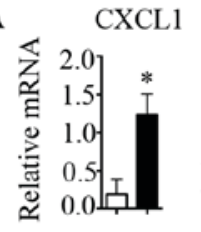

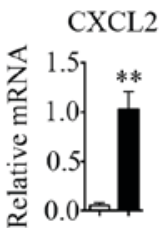
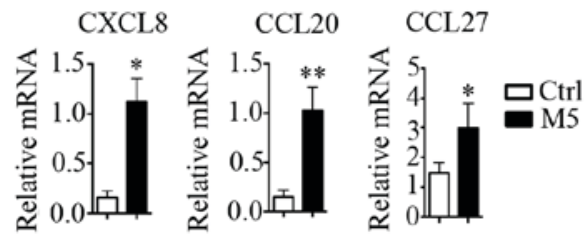

S100A8
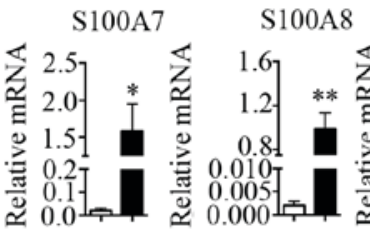

S100A9
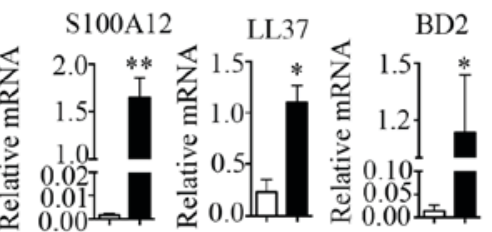

B

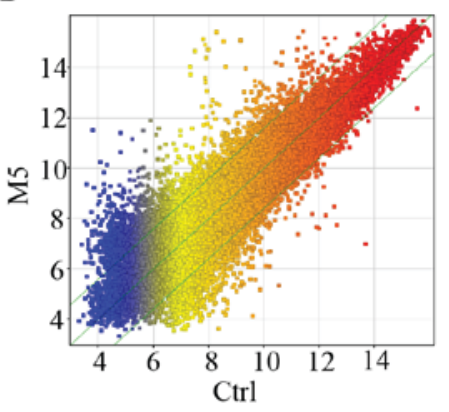

C

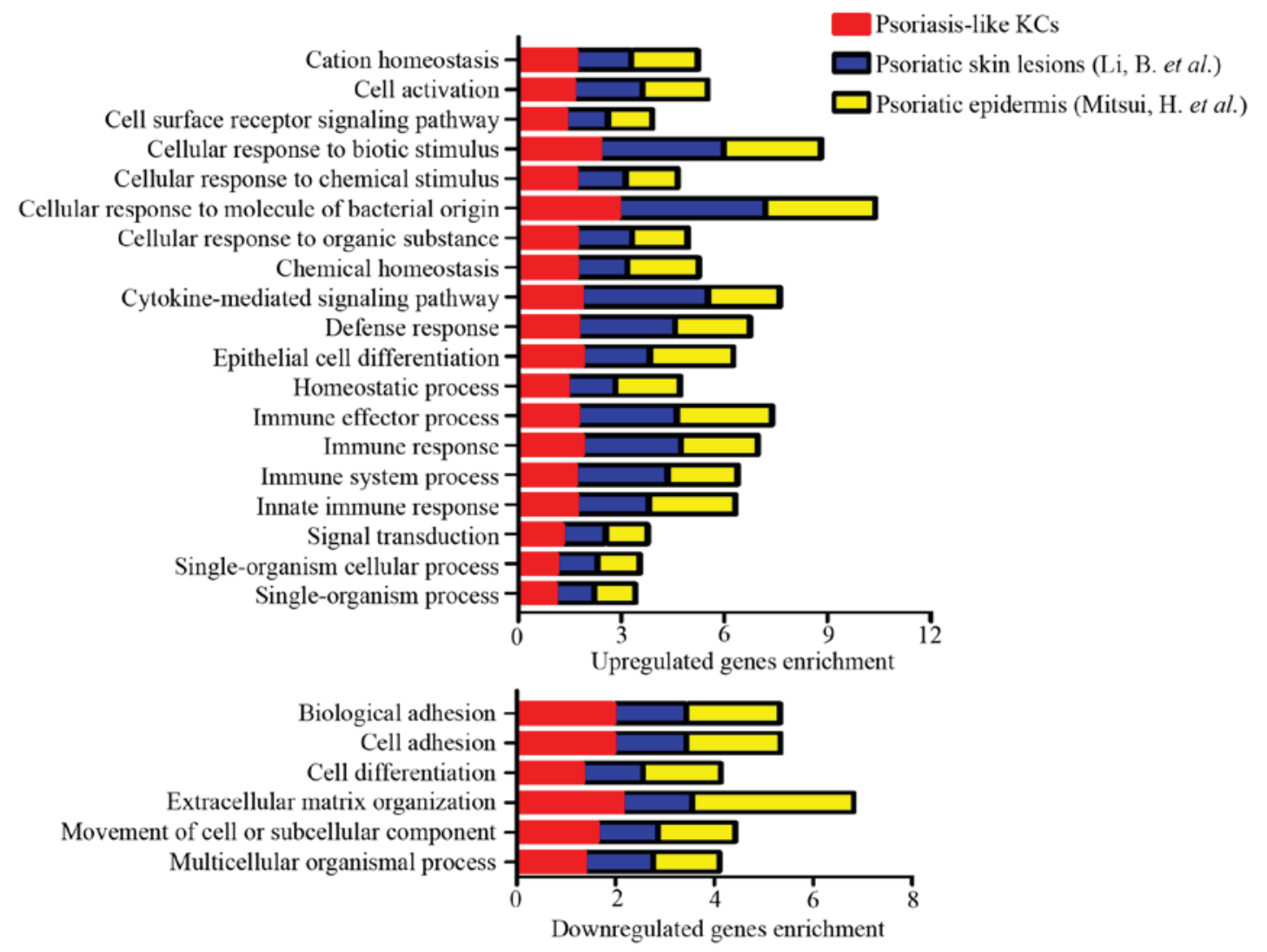

Figure 1. Comparison of transcriptomes in psoriasis-like KCs and patients with psoriasis. (A) KCs were stimulated with M5 for $24 \mathrm{~h}$, reverse transcription-quantitative polymerase chain reaction analysis was performed for each indicated gene. (B) Whole transcriptome gene expression analysis visualizing the variation in the expression between M5 and control groups. Different colors represent gene expression levels. The values on the $\mathrm{X}$ and $\mathrm{Y}$ axes in the scatter plot are the averaged $\log 2$-scaled signal values of the groups. The green lines showed a fold change of 3.0. The levels of the mRNAs above the top green line and below the bottom green line were $>3.0$-fold different between the two groups. (C) DEGs of psoriasis-like KCs, reported DEGs of psoriatic skin lesions by Li et al (18), and reported DEGs of psoriatic epidermis by Mitsui et al (19), were separately sorted according to biological process. Enrichment analysis was performed using Gorilla and $\mathrm{P}<0.001$ was the enrichment significance threshold. Bars represent different biological processes. Data are presented as the mean \pm standard deviation, $\mathrm{n}=3,{ }^{*} \mathrm{P}<0.05,{ }^{* *} \mathrm{P}<0.01$ vs. Ctrl group. DEGs, differentially expressed genes; CXCL, C-X-C motif chemokine ligand; CCL, C-C motif chemokine ligand; Ctrl, control; BD, $\beta$-defensin; KCs, keratinocytes.

epidermis vs. non-lesional psoriatic epidermis (19)]. A core set of 506 overlapping genes $(329+100+77)$ were identified, which were not only differently expressed in psoriasis-like $\mathrm{KCs}$, but also in psoriatic lesions or lesional epidermis of patients with psoriasis (Fig. 2, step 2). Thus, 506 psoriasis disease-associated genes were obtained.

In order to screen for novel psoriasis-associated genes, an additional screening was performed to overlap between the 506 and the known psoriasis-associated genes from the GeneCards database. In the 506 psoriasis disease-associated genes, 73 psoriasis-associated genes that had been well described in previous studies according to GeneCards database were excluded (Fig. 2, step 3), and the remaining 433 genes (Fig. 2, step 3, marking i) were selected as novel psoriasis disease-associated genes.

If the candidate genes were present in the psoriasis susceptibility region, which genetic loci were also linked to psoriasis as described in the 'Materials and methods' section, these genes were likely to serve an important role in psoriasis. More than 100 genetic loci linked to psoriasis were obtained from OMIM (www.omim.org/search?inde $\mathrm{x}=$ entry\&search $=$ psoriasis\&filter $=\mathrm{gm} \_$exists\%3Atr ue\& 
Step 1 Transcriptome

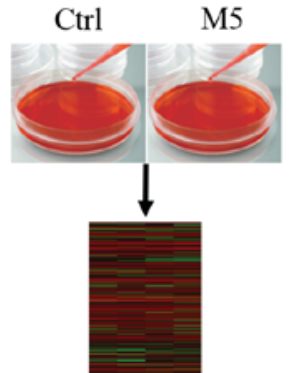

DEGs in psoriasis-like $\mathrm{KCs}$, $\mathbf{n}=\mathbf{2 9 5 7}(\mathrm{FC}>3, P<0.01)$

Step 2

\section{Overlap with DEGs in} psoriasis patients

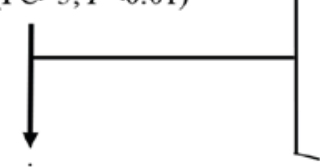

Overlapping genes

$\mathrm{n}=506$

Step 3

Exclude the psoriasis-associated genes of GeneCards database

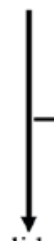

New candidate genes $\mathrm{n}=\mathbf{4 3 3}$

Step 4

Genetic loci linked to psoriasis

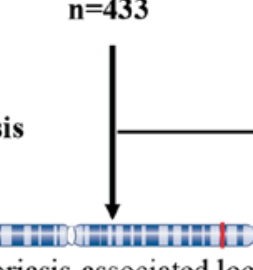

Psoriasis-associated loci

harbored genes

$$
\mathrm{n}=\mathbf{1 6 5}
$$

Step 5

EST sources

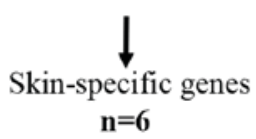

Figure 2. Screening of key skin-specific candidate genes from transcriptome of psoriasis-like KCs. Step 1: 2,957 DEGs were identified in psoriasis-like KCs. Step 2: Using the overlap between the 2,957 DEGs in psoriasis-like KCs and the reported DEGs in psoriasis patients [DEGs from Li et al (18) and by Mitsui et al (19)], and 506 overlapping genes were identified. Red number represents selected overlapping genes. Step 3: Further screening was performed to overlap between the 506 genes and the psoriasis-associated genes from GeneCards database excluding 73 psoriasis-associated genes. Step 4: In an additional screening was performed by genetic locus retrieval, and 165 genes were located near summary genetic loci linked to psoriasis. Step 5: These 165 genes were identified by analyses expression patterns in multiple human tissues inferred from EST sources. Finally, six genes were identified as skin-specific candidate genes. Ctrl, control; DEGs, differentially expressed genes; KCs, keratinocytes; FC, fold change; EST, expressed sequence tag.

sort=chromosome_number+asc $\% 2 \mathrm{C}+$ chromosome_sort+asc $\&$ start $=1 \&$ limit $=100$ \& retrieve $=$ geneMap) and from previous studies (20-29). These 433 novel psoriasis-associated genes were further screened by genetic locus retrieval, and it was revealed that 165 genes were located near the summary genetic loci linked to psoriasis (Fig. 2, step 4, marking ii), and these were identified as key novel psoriasis-associated genes.

Screening of skin-specific candidate genes associated with psoriasis. To screen skin-specific genes from the 433 novel psoriasis-associated genes, an additional screening was performed to assess expression patterns of 165 genes in normal human tissue by referring to the EST resources. Based on the TPM value of indicted gene in various tissues, six genes (EREG, NIPAL4, PLA2G4E, SERPINB7, SLC1A6,
$W F D C 12)$ that were relatively tissue-specific and highly expressed in human skin were selected (Fig. 2, step 5). The tissue expression pattern of these six genes was demonstrated by their EST profile and the heat map analysis of $\log 2-\mathrm{FC}$ of TPM (Fig. 3).

As shown in Fig. 2, an integrated approach was used, combining psoriasis transcriptome data derived from the GeneCards database, psoriasis-associated locus and EST resources. Through the above screening process, six significantly differentially expressed genes (EREG, NIPAL4, PLA2G4E, SERPINB7, SLC1A6 and WFDC12) in psoriatic $\mathrm{KCs}$ and lesional skin/epidermis of patients with psoriasis were identified. As novel psoriasis-associated candidate genes, these six genes were located near the psoriasis-associated locus, and they were relatively tissue-specific and highly expressed in human skin tissue. 


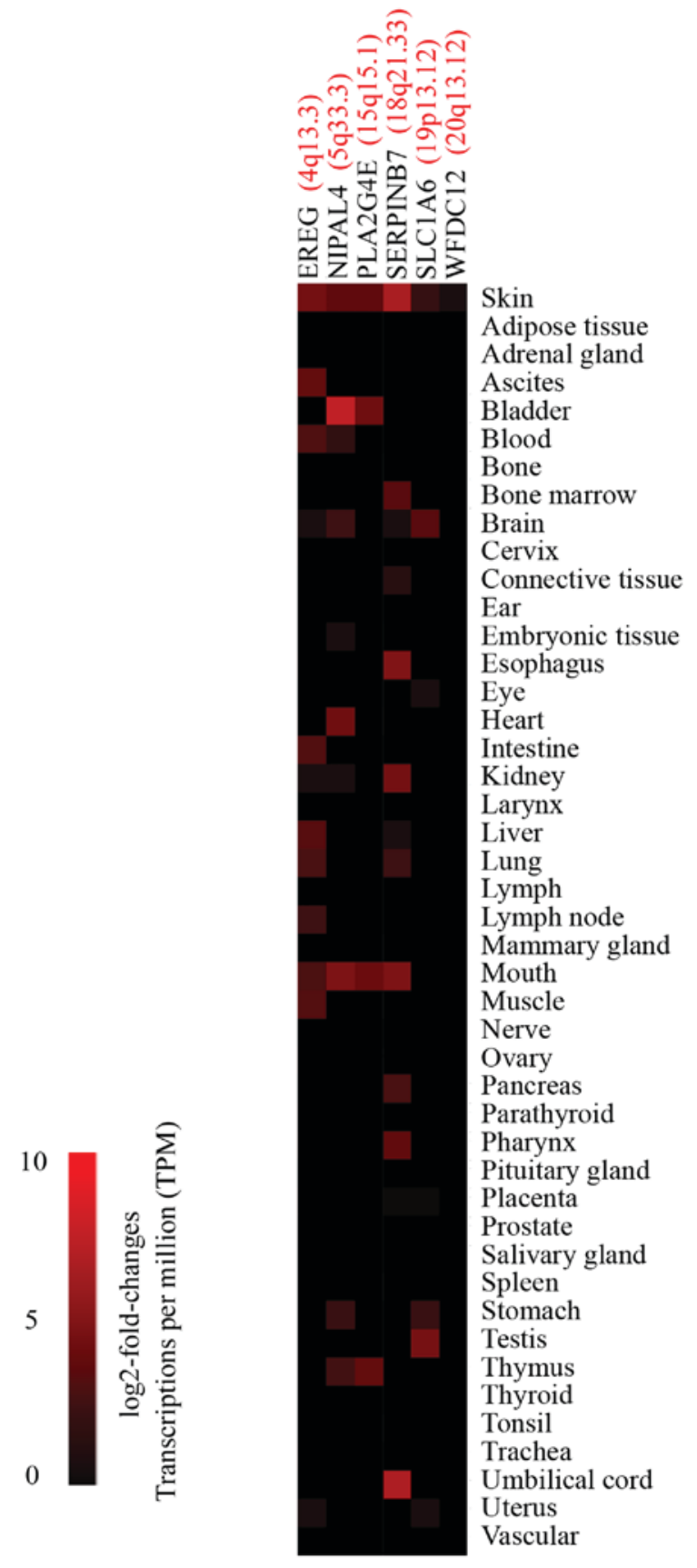

Figure 3. EST profiles of screened skin-specific candidate genes. Heat map analysis of the differential expression of six skin-specific candidate genes in normal human tissues. Black, no expression; red, high expression. Color gradient, $\log 2$-fold-changes of transcriptions per million from EST sources. Red indicates the genetic loci linked to psoriasis. EST, expressed sequence tag; EREG, epiregulin; NIPAL4, NIPA like domain containing 4; PLA2G4E, phospholipase A2 group IVE; SERPINB7, serpin family B member 7; SLC1A6, solute carrier family 1 member 6; WFDC12, WAP four-disulfide core domain 12 .

Identification of candidate genes specifically and highly expressed in mouse skin epidermis. The tissue expression profile of the six novel candidate genes in the mouse was confirmed by RT-qPCR, and the results showed that four genes (EREG, NIPAL4, SERPINB7 and WFDC12) were highly expressed in the skin, but their expression levels were lower in other major organs and tissues, including the large intestine, lung, liver, testis, ovary, brain, spleen, and kidney, small intestine and heart (Fig. 4A). This result was consistent with their EST profiles (Fig. 3). However, PLA2G4E and SLC1A6 exhibited no such skin specificity (Fig. 4A).

The epidermis is composed primarily of $\mathrm{KCs}$ that are key skin-specific immune cells. The dermis is primarily composed of other immunocyte cell types, such as fibroblasts, dendritic cells (DCs), T helper cells, $\gamma \delta \mathrm{T}$ cells $(1,2)$. EREG, NIPAL4, SERPINB7 and WFDC12 exhibited higher expression levels in mouse epidermis than in dermis (Fig. 4B). This result suggested that these genes exhibited higher expression levels in the key skin-specific immune cells (KCs) than in other dermis immunocyte cell types, indicating their skin specificity.

Identification of candidate genes that are specifically increased in psoriasis-like skin epidermis. In order to define whether the candidate genes were differentially regulated in psoriasis-like skin, mRNA from IMQ-treated or untreated dorsal skin (day 0) was isolated and analyzed by RT-qPCR. Upon treatment with IMQ, the four candidate genes (EREG, NIPAL4, SERPINB7 and WFDC12) were strongly induced on day 4 (EREG, NIPAL4, SERPINB7) and day 6 (WFDC12) (Fig. 5A). This result suggested that these four candidate molecules may be involved in local immune responses in psoriatic skin tissue.

Subsequently, in order to evaluate the psoriatic skin specificity of the four candidate genes, mRNA expression levels in dissected epidermis and dermis of IMQ-treated dorsal skin or normal dorsal skin was analyzed by RT-qPCR. In normal skin and IMQ-treated skin, the four genes were all highly expressed in epidermis compared with the dermis (Fig. 5B). Furthermore, the mRNA expression levels of EREG, NIPAL4 and SERPINB7 after 4 days, and WFDC12 after 6 days of IMQ treatment were dramatically increased in the epidermis of dorsal skin. However, in dermis, there was no significant difference in the expression levels of these four genes between normal and IMQ-treated skin. As immunocytes are recruited to the dermis in psoriasis, this result indicated that the expressions levels of these molecules were differentially regulated in psoriatic epidermis (which is predominantly $\mathrm{KCs}$ ), but not in immunocytes that infiltrate the psoriatic dermis. In addition, as many important immune molecules may exhibit expression alteration in peripheral blood immunocytes, published microarray data (GSE40263) of peripheral blood mononuclear cells (PBMCs) from patients with psoriasis, was used in the current study. There was no significant difference in the PBMCs expression levels of these four genes in the psoriasis and healthy control groups (Fig. 5C). Combined with differences in expression of these four genes induced by M5 in the psoriasis-like $\mathrm{KC}$ model (Fig. 5C), these results suggested that the expression of EREG, NIPAL4,SERPINB7 and WFDC12 were specifically regulated and highly expressed in psoriatic KCs (psoriatic skin-specific immune cells), but not in the dermis or PBMCs involved in the local immune response to psoriasis. Thus, these genes also were novel psoriatic skin-specific genes, and may serve a unique role in the pathogenesis of psoriasis, but this needs to be investigated further.

Differential expression pattern of candidate genes in SIRS. In order to confirm whether the candidate genes were involved in local immune responses in the skin, the expression levels of the five genes (TNF- $\alpha, E R E G, N I P A L 4$, SERPINB7 and 

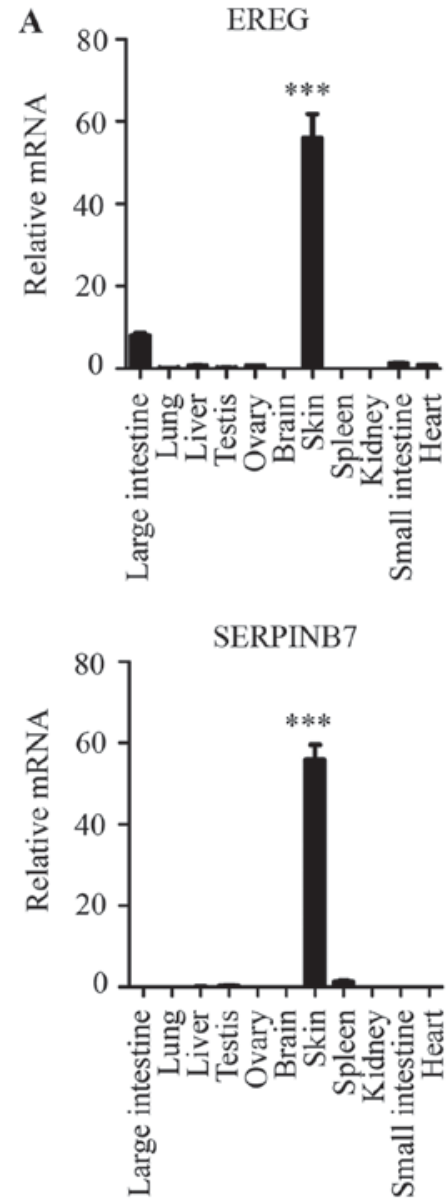
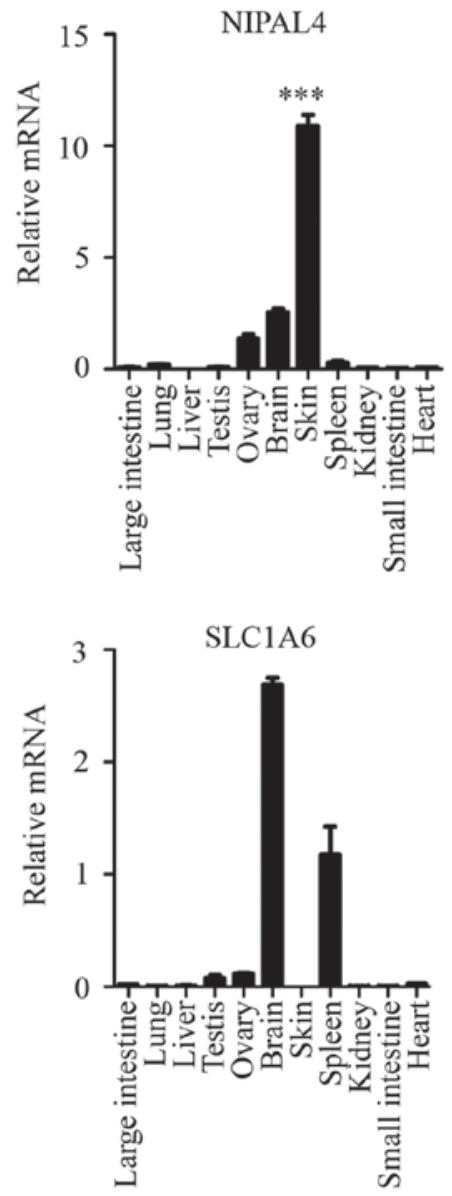
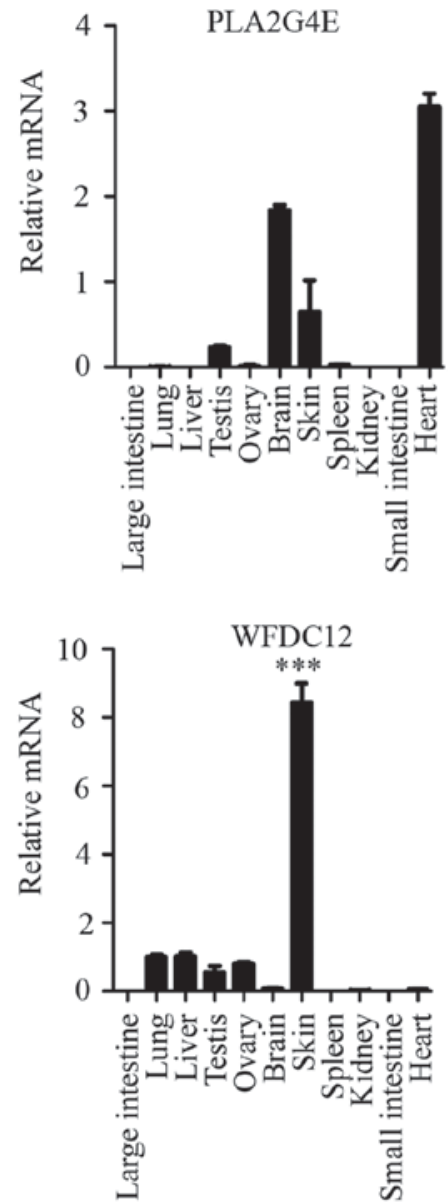

B

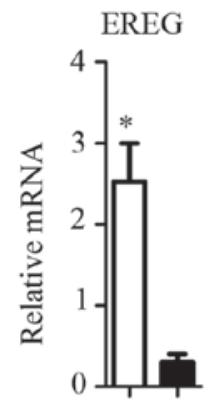

NIPAL4

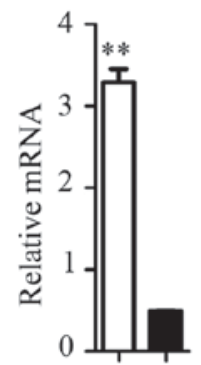

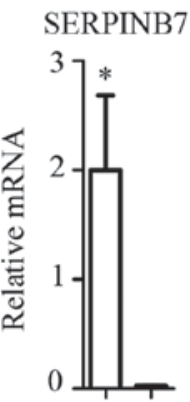

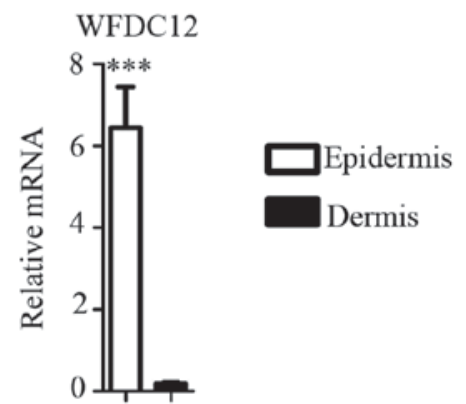

Figure 4. Identification of key skin-specific candidate genes. (A) RT-qPCR analysis was performed for each indicated gene from C57BL/6 WT mice different tissues. Data presented the mean \pm standard deviation of three independent experiments, $n=5$ mice, ${ }^{* * *} \mathrm{P}<0.001$ skin vs. the other major organs and tissues (large intestine, lung, liver, testis, ovary, brain, spleen, and kidney, small intestine and heart). (B) RT-qPCR analysis was performed for each indicated gene from dissected mice epidermis and dermis. Data presented the mean \pm standard deviation of three independent experiments, $n=5$ mice, ${ }^{*} \mathrm{P}<0.05,{ }^{* * *} \mathrm{P}<0.01$, ${ }^{* * * *} \mathrm{P}<0.001$ vs. dermis. RT-qPCR, reverse transcription-quantitative polymerase chain reaction; EREG, epiregulin; NIPAL4, NIPA like domain containing 4; PLA2G4E, phospholipase A2 group IVE; SERPINB7, serpin family B member 7; SLC1A6, solute carrier family 1 member 6; WFDC12, WAP four-disulfide core domain 12 .

WFDC12) were determined by RT-qPCR in the LPS-induced SIRS and normal saline group. There was no difference in expression of the five genes in skin tissue between the two groups, but significantly higher levels of TNF- $\alpha$ were detected in the large intestine, lung, liver, testis, ovary, brain, spleen, kidney, small intestine, and heart of the SIRS group compared with the saline-treated control. These results suggested that LPS can induce inflammatory response of numerous tissues, but not in the skin in the SIRS model. Furthermore, it was found that the expression levels of EREG in lung, kidney, small intestine and heart of the SIRS group were higher compared with those of normal saline group. The expression levels of NIPAL4 in lung and small intestine were significantly higher in the SIRS model compared with the control mice. WFDC12 exhibited higher expression in lung, liver and heart of the SIRS model compared with those of the control mice. By contrast, there was no significant difference in the SERPINB7 expression levels in all tissues between the two groups. This result suggested that SERPINB7 may not participate in the local immune responses in the various tissues (large intestine, lung, liver, testis, ovary, brain, spleen, kidney, small intestine and heart) of the SIRS model examined (Fig. 6). 

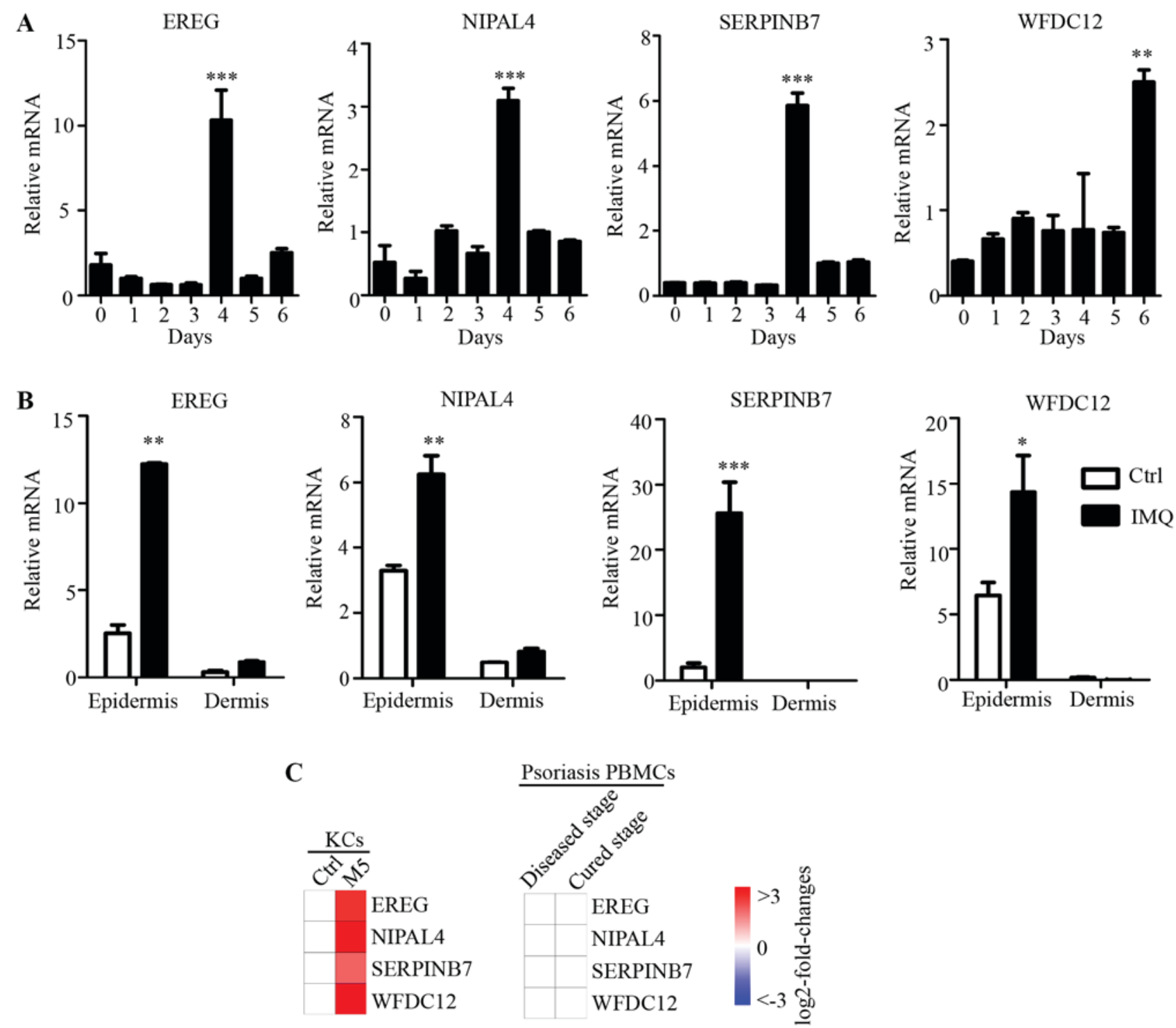

Figure 5. Expression of key candidate genes specifically increased in psoriatic epidermis. (A) The dorsal skin of C57BL/6 ( $=5$ ) mice was treated daily with IMQ $(55 \mathrm{mg}$ ) for 6 days, RT-qPCR analysis was performed for each indicated gene at day 0,1,2,3,4,5 and 6 . Data are presented the mean \pm standard deviation of three independent experiments, ${ }^{* * * *} \mathrm{P}<0.001,4$ day vs. other groups; ${ }^{* *} \mathrm{P}<0.01,6$ day vs. other groups. (B) The dorsal skin of the $\mathrm{C} 57 \mathrm{BL} / 6(\mathrm{n}=5)$ mice were treated daily with IMQ $(55 \mathrm{mg})$ for 6 days, then the dermis and epidermis were obtained by digested separation. RT-qPCR analysis of $E R E G$, NIPAL4 and SERPINB7 at 4 days after IMQ treatment, WFDC12 at 6 days after IMQ treatment. Data are presented the mean \pm standard deviation of three independent experiments, ${ }^{*} \mathrm{P}<0.05,{ }^{* *} \mathrm{P}<0.01,{ }^{* * *} \mathrm{P}<0.001$ vs. Ctrl. (C) Heat map of four key candidate genes in psoriasis-like KCs and psoriasis PBMCs from published microarray data (GSE40263). White, no change; green, downregulated; red, upregulated. Color gradient, log2-fold-changes. RT-qPCR, reverse transcription-quantitative polymerase chain reaction; EREG, epiregulin; NIPAL4, NIPA like domain containing 4; SERPINB7, serpin family B member 7; WFDC12, WAP four-disulfide core domain 12; SLC1A6, solute carrier family 1 member 6; Ctrl, control; IMQ, imiquimod; KC, keratinocytes; PBMCs, peripheral blood mononuclear cells.

SERPINB7 mRNA expression levels in lesional and non-lesional psoriatic skin of patients with psoriasis. mRNA from lesional and non-lesional psoriatic skin of patients with psoriasis was isolated and was examined by RT-qPCR. The mRNA expression level of SERPINB7 (selected as it was involved in the activated immune response of the skin, not other tissues) in lesional psoriatic skin of patients with psoriasis was significantly higher than in non-lesional psoriatic skin of the same patients (Fig. 7).

\section{Discussion}

Psoriasis is a common skin disease affecting $2 \%$ of the population worldwide and is characterized by increased proliferation and abnormal differentiation of KCs (33). There are not many effective psoriatic skin-specific targeted drugs to control the symptoms. In the present study, DEGs were detected from KCs (a skin-specific immune cell type), derived from psoriatic and non-psoriatic human and mice tissues, from a SIRS model, and skin-specific genes were identified. The present study introduced a cutaneous tissue-specific target for skin-related diseases treatment and at the same time provided a novel method for the exploration of unknown cutaneous tissue-specific targets for disease treatment.

Many DEGs identified in previous studies were localized to defined lesional psoriatic skin, or the epidermis and dermis of psoriatic lesions $(18,19)$, whereas expression pattern analysis 

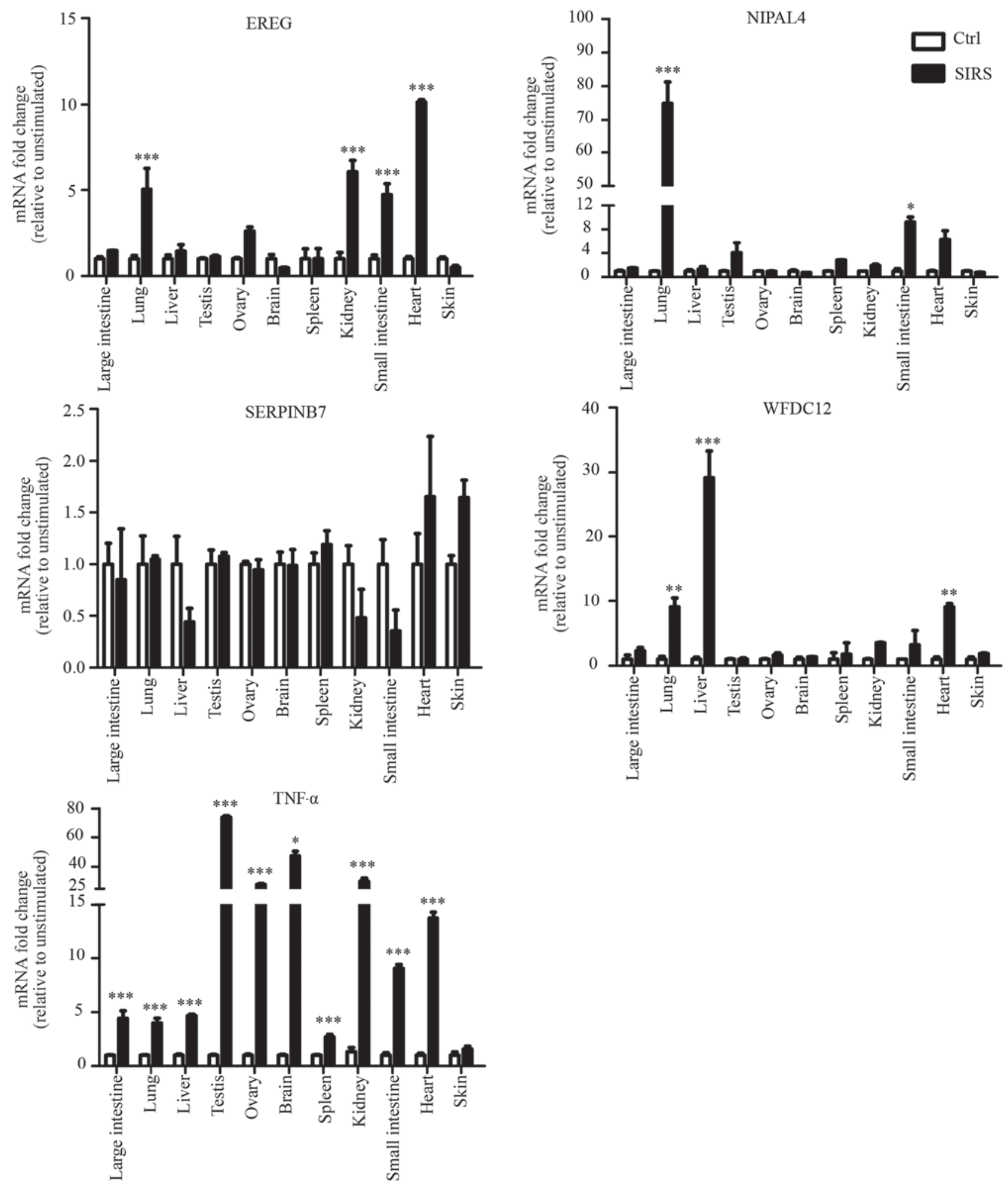

Figure 6. Differential expression patterns of candidate genes in SIRS. Tissue expression patterns of psoriatic-specific candidate genes in lipopolysaccharide-induced SIRS and normal saline group $(\mathrm{n}=5$, each). Reverse transcription-quantitative polymerase chain reaction analysis of the indicated genes in various tissues was performed, and data presented the mean \pm standard deviation of three independent experiments, ${ }^{*} \mathrm{P}<0.05,{ }^{* * *} \mathrm{P}<0.01,{ }^{* * * *} \mathrm{P}<0.001$ vs. the Ctrl group. EREG, epiregulin; NIPAL4, NIPA like domain containing 4; Ctrl, control; SIRS, systemic inflammatory response syndrome; SERPINB7, serpin family B member 7; WFDC12, WAP four-disulfide core domain 12; TNF $\alpha$, tumor necrosis factor- $\alpha$.

in a psoriasis-like KCs model has not been reported frequently. In the present study, the gene expression profile was produced using a microarray analysis of psoriasis-like KCs. Although there have been data sets produced single cytokine-induced gene expression changes in cultured KCs $(34,35)$, it appears that single cytokine stimulation has a limited effect on KCs, namely, a limited number and a limited modulated expression of targeted genes, reflecting only partial features of psoriasis (16). In previously published transcriptional profiling experiments, gene sets for $\mathrm{KC}$ responses to cytokines involved 


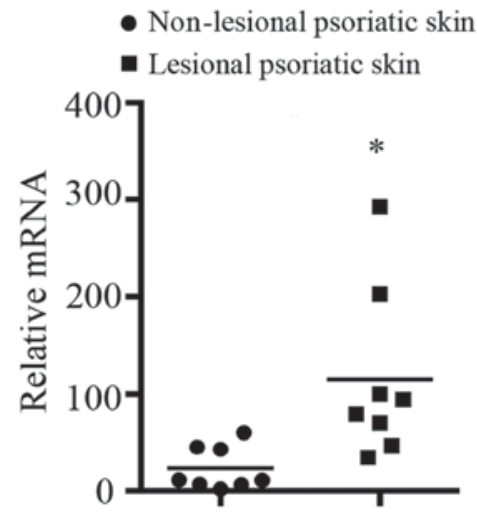

Figure 7. SERPINB7 mRNA expression levels in lesional and non-lesional psoriatic skin of patients with psoriasis. Reverse transcription-quantitative polymerase chain reaction analysis was performed for SERPINB7 in lesional and non-lesional psoriatic skin of patients with psoriasis. Data are presented as the mean \pm standard deviation, $\mathrm{n}=8,{ }^{*} \mathrm{P}<0.05$ vs. non-lesional psoriatic skin. SERPINB7, serpin family B member 7.

in psoriasis were curated, including IL-17, TNF- $\alpha$, and IL-22, alone and as a combination (36-39). In the present study, a psoriasis-like KCs model was established by treatment with IL-17, IL-1 $\alpha$, IL-22, TNF- $\alpha$ and oncostatin-M, which produced a strong transcriptional effect on KCs chemokines, cytokines, and antimicrobial peptide production, and these cells exhibit a psoriasis-like profile $(16,40)$. In the present study, by comparing transcriptomes of psoriasis-like KCs and previously published data sets of lesional psoriatic skin/epidermis, it was revealed that enriched functions of DEGs had highly similar patterns in the current study and the previously published data. Thus, psoriatic skin-specific genes were identified by analysis of DEGs in psoriasis-like KCs.

Psoriasis is a complex multifactorial disease and the development of this disease remains largely unexplored. Recent research identified psoriasis susceptibility loci and genes that are closely associated with the pathogenesis of psoriasis (5). Genome-wide linkage scans and high throughput studies have been used to identify genes responsible for familial psoriasis and several susceptibility loci (41). In the present study, an integrated approach was used, which may be helpful for exploring the contributory factors involved in the initiation of a complex multifactorial disease such as psoriasis.

ESTs derived from different cDNA libraries can be prepared from different tissues, organs or cell types. It provides a rapid and efficient approach for deciphering gene expression levels in different tissues and screening tissue-specific molecules $(42,43)$. Previous studies have used EST data to identify tissue-specific genes in the human prostate (44), heart (45), retina (46) and in cancer tissue (47). These studies highlight the advantages of using this approach. In the present study, integrated analysis of human ESTs provided a robust platform for psoriasis-like KCs transcriptome screening. Four genes with skin-specific expression were identified, indicating that EST assessment was highly accurate.

Gerber et al (48), using the Body Index of Human Gene Expression database and comparing the ratio of mean gene expression in skin with other tissue/cell types, investigated eight genes out of the top 100 genes preferentially expressed in normal human skin. The expression profiles of these eight candidate genes (mucin like 1, WFDC5, SERPINB7, chromosome 5 open reading frame 46, transmembrane protein $45 A$, $G$ protein-coupled receptor 115 , cadherin related family member 1 and $G$ protein-coupled receptor 87$)$ were analyzed in five tissues (skin, spleen, kidney, brain, liver) and four cell types (keratinocytes, fibroblasts, PBMCs, endothelial cells). The expression levels in cytokine-stimulated keratinocytes and in biopsies of skin diseases were analyzed (48). In the present study, novel candidate genes were investigated in another manner. Initially, based on gene description and publications from the human gene database (GeneCards), 73 genes that were closely associated with psoriasis were excluded. Subsequently, based on the summary of genetic loci linked to psoriasis, 165 candidate genes were identified. Finally, as skin specific cells, KCs constitute the majority of cells in the skin's epidermis; therefore, the expression of the six candidate genes (EREG, NIPAL4, PLA2GE, SERPINB7, SLC1A6 and $W F D C 12)$ was examined in epidermis and dermis samples form mice.

The skin is composed of two distinct regions, the epidermis and dermis, it contains a variety of immune cells $(1,2)$. The $\mathrm{KCs}$ are the predominant cell type in the epidermis, it is highly specialized epithelial cells designed to perform a very specific function. The dermis contains cells of the immune system including T cells and DCs $(1,2,4)$. In the normal condition, four candidate genes (EREG, NIPAL4, SERPINB7 and WFDC12) were highly expressed in skin compared with other tissues. In skin tissue, the expression of these genes was higher in the epidermis than in the dermis. This suggested that the four candidate genes were highly expressed in KCs. KCs exhibit hyperproliferation and abnormal differentiation in psoriatic epidermis, and a large number of inflammatory cells infiltrate into the dermal lesions $(5,6)$. The expression levels of the four candidate genes were increased in psoriasis-like skin lesion. Furthermore, it was revealed that the expression levels of these candidate genes were increased in psoriatic epidermis compared with normal controls; however, the levels were not different in psoriatic dermis compared with normal controls. Furthermore, in psoriasis-like KCs, the expression levels of these genes were increased compared with untreated KCs. This suggested that the four candidate genes were highly expressed in psoriasis-like $\mathrm{KCs}$, and they may be involved in the local immune response of psoriatic KCs, suggesting that they have psoriatic skin-specific roles.

The pathophysiology of SIRS involves a systemic immune response that affects pulmonary, gastrointestinal and renal function. As LPS induced the systemic expression of the proinflammatory cytokine TNF- $\alpha$ in various tissues (large intestine, lung, liver, testis, ovary, brain, spleen, kidney, small intestine and heart), the systemic inflammatory response was activated. However, TNF- $\alpha$ and the four other detected genes (EREG, NIPAL4, SERPINB7 and WFDC12) exhibited no difference in expression the skin between the SIRS and control group. This suggested that LPS may have not induced an inflammatory response in skin tissue in the SIRS model. EREG, NIPAL4, and WFDC12 were upregulated in certain tissues in the SIRS model compared with the normal control. There was no significant difference in SERPINB7 expression between the two groups in the all tissues analyzed. These results suggested that SERPINB7 was not involved in the 
activated immune response of various tissues, except for the skin. SERPINB7, a serpin peptidase inhibitor, has critical roles in the immune system; it can increase mesangial cell proliferation and extracellular matrix (ECM) deposition and markedly suppress cell motility and invasion (49). SERPINB7 appears to be involved in maintaining tissue integrity by preserving ECM homeostasis, and loss of expression may lead to loss of cell adhesion and tissue integrity (49). SERPINB7 exhibits substantial expression variation in skin disorders, such as palmoplantar keratosis (50). Additionally, the mRNA levels of SERPINB were significantly higher in lesional psoriatic skin than in non-lesional psoriatic skin of patients with psoriasis. Therefore, SERPINB7 requires further investigation to clarify its potential role in the pathogenesis of psoriasis.

As novel psoriatic skin-specific genes, EREG, NIPAL4 and $W F D C 12$ may also be valuable candidates for further exploration. It was previously reported that EREG and WFDC12 serve the critical immuno-regulatory roles in skin. EREG encodes epiregulin, which is a secreted peptide hormone and member of the epidermal growth factor family of proteins, is overexpressed in psoriatic epidermis (51). Functions associated with this gene include growth factor activity and epidermal growth factor receptor binding. Secreted epiregulin induces downregulation of inflammatory cytokine IL-18 mRNA expression in KCs (52) and can also stimulate proliferation of KCs. WFDC12 is one of an 18-member family of secreted proteins reported as protease inhibitors, and is an antimicrobial peptide also reported to participate in inflammation and host defense (53). However, the expression of EREG and WFDC12 in psoriatic skin lesions is currently unknown. In addition, there are few studies on the role of NIPAL4 in the skin. NIPAL4, also known as ichthyin, is composed of several transmembrane domains. It is associated with keratins and desmosomes in $\mathrm{KCs}$ and is involved in lipid metabolism $(54,55)$. Mutations in this gene have been associated with autosomal recessive congenital ichthyosis (56).

The present data strongly suggests that these genes (EREG, NIPAL4, SERPINB7 and WFDC12) were specifically expressed in psoriatic skin, and they may be involved in the local immune response of psoriatic skin. Further studies are required to gain further insights into their regulation and potential role in skin disorders.

In the present study, a pathological tissue-specific molecular screening method was established. This method used psoriatic transcriptome data, psoriasis-associated gene information, genetic loci associated with psoriasis and normal/pathological tissue expression patterns. Through the screening procedure, candidate genes with genetic loci associated with psoriasis that were specific expressed in normal skin and exhibited high expression in psoriatic $\mathrm{KCs}$, rather than in other inflammatory cells or tissues, were identified. The present study identified the potential key pathogenic tissue-specific molecules for diseases, and the psoriatic skin-specific genes (EREG, NIPAL4, SERPINB7 and WFDC12) may represent potential biomarkers or drug targets for the development of future diagnostics/therapeutics to treat psoriasis.

\section{Acknowledgements}

The authors thanks to our research assistants, Ms Wenling Wu and Mr Yifan Zhou (Department of Biotherapy, Cancer
Center/Collaborative Innovation Center for Biotherapy, West China Hospital, Sichuan University) for their technical assistance.

\section{Funding}

The present study was supported by grants from the National Natural Science Foundation of China (grant nos. 31271483, 81703132 , 81472650, 81673061, 81573050, 31872739, 81602763 and 8160070706), the China Postdoctoral Science Foundation funded project (grant no. 2018M631087), the National Science and Technology Major Project (grant nos. 2018ZX09303006-001-006 and 2018ZX09201004-003, 2012ZX10002006-003-001 and 2013ZX09301304001 -003), the Sichuan Provincial Outstanding Youth Fund (grant no. 2015JQ0025).

\section{Availability of data and materials}

The analyzed data sets generated during the present study are available from the corresponding author on reasonable request.

\section{Authors' contributions}

$\mathrm{ZW}$ and JL designed the experiments and the present study. ZW, HPZ, HZ, NH, XW and JZ collected data and did experiments. ZW, XL, XT, ZH, XZ and WL analyzed the data. ZW, HPZ, HZ, XW and JZ contributed to critical revisions of the text.

\section{Ethics approval and consent to participate}

The present study was performed in accordance with the principles of the Helsinki Declaration and approved by the Ethics Committee of the West China Hospital, Sichuan University. Written informed consent was obtained from all study participants prior to the present study. The animal protocols were approved by the Committee on the Ethics of Animal Experiments of the Sichuan University (Chengdu, China). The experimental procedures were conducted according to the ethical guidelines for the care and use of laboratory animals of the National Institutes of Health and the IASP.

\section{Patient consent for publication}

Not applicable.

\section{Competing interests}

The authors declare that they have no competing interests.

\section{References}

1. Nestle FO, Di Meglio P, Qin JZ and Nickoloff BJ: Skin immune sentinels in health and disease. Nat Rev Immunol 9: 679-691, 2009.

2. Heath WR and Carbone FR: The skin-resident and migratory immune system in steady state and memory: Innate lymphocytes, dendritic cells and T cells. Nature Immunol 14: 978-985, 2013.

3. Boehncke WH and Schön MP: Psoriasis. Lancet 386: 983-994, 2015. 
4. Nestle FO, Kaplan DH and Barker J: Psoriasis. NEngl J Med 361: 496-509, 2009.

5. Lowes MA, Suárez-Fariñas M and Krueger JG: Immunology of psoriasis. Annu Rev Immunol 32: 227-255, 2014

6. Bos JD, de Rie MA, Teunissen MB and Piskin G: Psoriasis: Dysregulation of innate immunity. Br J Dermatol 152: 1098-1107, 2005

7. Perera GK, Di Meglio P and Nestle FO: Psoriasis. Annu Rey Pathol 7: 385-422, 2012

8. Kim J and Krueger JG: The immunopathogenesis of psoriasis. Dermatol Clin 33: 13-23, 2015

9. Crow JM: Therapeutics: Silencing psoriasis. Nature 492 (Suppl): S58-S59, 2012

10. Williams SC: New biologic drugs get under the skin of psoriasis Nat Med 18: 638, 2012.

11. Liu X, Fang L, Guo TB, Mei H and Zhang JZ: Drug targets in the cytokine universe for autoimmune disease. Trends Immunol 34: 120-128, 2013

12. Iborra M, Beltrán B, Bastida G, Aguas M and Nos P: Infliximab and adalimumab-induced psoriasis in Crohn's disease: A paradoxical side effect. J Crohns Colitis 5: 157-161, 2011.

13. Matos TR, O'Malley JT, Lowry EL,Hamm D, Kirsch IR, Robins HS Kupper TS, Krueger JG and Clark RA: Clinically resolved psoriatic lesions contain psoriasis-specific IL-17-producing $\alpha \beta$ T cell clones. J Clin Invest 127: 4031-4041, 2017.

14. Leonardi C, Matheson R, Zachariae C, Cameron G, Li L, Edson-Heredia E, Braun D and Banerjee S: Anti-interleukin-17 monoclonal antibody ixekizumab in chronic plaque psoriasis. $\mathrm{N}$ Engl J Med 366: 1190-1199, 2012.

15. Langley RG and Ellis CN: Evaluating psoriasis with psoriasis area and severity index, psoriasis global assessment, and lattice system physician's global assessment. J Am Acad Dermatol 51: 563-569, 2004

16. Guilloteau K, Paris I, Pedretti N, Boniface K, Juchaux F, Huguier V, Guillet G, Bernard FX, Lecron JC and Morel F: Skin inflammation induced by the synergistic action of IL-17A, IL-22, oncostatin M, IL-1\{alpha\}, and TNF-\{alpha\} recapitulates some features of psoriasis. J Immunol 184: 5263-5270, 2010.

17. Teng X, Hu Z, Wei X, Wang Z, Guan T, Liu N, Liu X, Ye N, Deng G, Luo C, et al: IL-37 ameliorates the inflammatory process in psoriasis by suppressing proinflammatory cytokine production. J Immunol 192: 1815-1823, 2014.

18. Li B, Tsoi LC, Swindell WR, Gudjonsson JE, Tejasvi T, Johnston A, Ding J, Stuart PE, Xing X, Kochkodan JJ, et al: Transcriptome analysis of psoriasis in a large case-control sample: RNA-seq provides insights into disease mechanisms. J Invest Dermatol 134: 1828-1838, 2014.

19. Mitsui H, Suárez-Fariñas M, Belkin DA, Levenkova N Fuentes-Duculan J, Coats I, Fujita H and Krueger JG: Combined use of laser capture microdissection and cDNA microarray analysis identifies locally expressed disease-related genes in focal regions of psoriasis vulgaris skin lesions. J Invest Dermatol 132: 1615-1626, 2012

20. Genetic Analysis of Psoriasis Consortium \& the Wellcome Trust Case Control Consortium 2, Strange A, Capon F Spencer CC, Knight J, Weale ME, Allen MH, Barton A, Band G, Bellenguez C, et al: A genome-wide association study identifies new psoriasis susceptibility loci and an interaction between HLA-C and ERAP1. Nat Genet 42: 985-990, 2010.

21. Zhang XJ, Huang W, Yang S, Sun LD, Zhang FY, Zhu QX, Zhang FR, Zhang C, Du WH, Pu XM, et al: Psoriasis genome-wide association study identifies susceptibility variants within LCE gene cluster at 1q21. Nat Genet 41: 205-210, 2009.

22. de Cid R, Riveira-Munoz E, Zeeuwen PL, Robarge J, Liao W, Dannhauser EN, Giardina E, Stuart PE, Nair R, Helms C, et al: Deletion of the late cornified envelope LCE3B and LCE3C genes as a susceptibility factor for psoriasis. Nat Genet 41: 211-215, 2009.

23. Nair RP, Duffin KC, Helms C, Ding J, Stuart PE, Goldgar D, Gudjonsson JE, Li Y, Tejasvi T, Feng BJ, et al: Genome-wide scan reveals association of psoriasis with IL-23 and NF-kappaB pathways. Nat Genet 41: 199-204, 2009.

24. Sun LD, Cheng H, Wang ZX, Zhang AP, Wang PG, Xu JH, Zhu QX, Zhou HS, Ellinghaus E, Zhang FR, et al: Association analyses identify six new psoriasis susceptibility loci in the Chinese population. Nat Genet 42: 1005-1009, 2010.

25. Liu Y, Helms C, Liao W, Zaba LC, Duan S, Gardner J, Wise C, Miner A, Malloy MJ, Pullinger CR, et al: A genome-wide association study of psoriasis and psoriatic arthritis identifies new disease loci. PLoS Genet 4: e1000041, 2008.
26. Stuart PE, Nair RP,Ellinghaus E, Ding J, Tejasvi T, Gudjonsson JE, Li Y, Weidinger S, Eberlein B, Gieger C, et al: Genome-wide association analysis identifies three psoriasis susceptibility loci. Nat Genet 42: 1000-1004, 2010.

27. Gudjonsson JE, Ding J, Johnston A, Tejasvi T, Guzman AM, Nair RP, Voorhees JJ, Abecasis GR and Elder JT: Assessment of the psoriatic transcriptome in a large sample: Additional regulated genes and comparisons with in vitro models. J Invest Dermatol 130: 1829-1840, 2010.

28. Tsoi LC, Spain SL, Knight J, Ellinghaus E, Stuart PE, Capon F, Ding J, Li Y, Tejasvi T, Gudjonsson JE, et al: Identification of 15 new psoriasis susceptibility loci highlights the role of innate immunity. Nat Genet 44: 1341-1348, 2012.

29. Capon F, Bijlmakers MJ, Wolf N, Quaranta M, Huffmeier U, Allen M, Timms K, Abkevich V, Gutin A, Smith R, et al: Identification of ZNF313/RNF114 as a novel psoriasis susceptibility gene. Hum Mol Genet 17: 1938-1945, 2008.

30. Antal C, Teletin M, Wendling O, Dgheem M, Auwerx J and Mark M: Tissue collection for systematic phenotyping in the mouse. Curr Protoc Mol Biol Chapter 29: Unit 29A.4, 2007.

31. El Malki K, Karbach SH, Huppert J, Zayoud M, Reissig S, Schüler R, Nikolaev A, Karram K, Münzel T, Kuhlmann CR, et al: An alternative pathway of imiquimod-induced psoriasis-like skin inflammation in the absence of interleukin-17 receptor a signaling. J Invest Dermatol 133: 441-451, 2013.

32. Livak KJ and Schmittgen TD: Analysis of relative gene expression data using real-time quantitative PCR and the 2(-Delta Delta C(T)) method. Methods 25: 402-408, 2001

33. Greb JE, Goldminz AM, Elder JT, Lebwohl MG, Gladman DD, Wu JJ, Mehta NN, Finlay AY and Gottlieb AB: Psoriasis. Nat Rev Dis Primers 2: 16082, 2016.

34. Swindell WR, Xing X, Stuart PE, Chen CS, Aphale A, Nair RP, Voorhees JJ, Elder JT, Johnston A and Gudjonsson JE: Heterogeneity of inflammatory and cytokine networks in chronic plaque psoriasis. PLoS One 7: e34594, 2012.

35. Yano S, Banno T, Walsh R and Blumenberg M: Transcriptional responses of human epidermal keratinocytes to cytokine interleukin-1. J Cell Physiol 214: 1-13, 2008.

36. Wolk K, Kunz S, Witte E, Friedrich M, Asadullah K and Sabat R: IL-22 increases the innate immunity of tissues. Immunity 21 : 241-254, 2004

37. Banno T, Gazel A and Blumenberg M: Effects of tumor necrosis factor-alpha (TNF alpha) in epidermal keratinocytes revealed using global transcriptional profiling. J Biol Chem 279: 32633-32642, 2004.

38. Nograles KE, Zaba LC, Guttman-Yassky E, Fuentes-Duculan J, Suárez-Fariñas M, Cardinale I, Khatcherian A, Gonzalez J, Pierson KC, White TR, et al: Th17 cytokines interleukin (IL)-17 and IL-22 modulate distinct inflammatory and keratinocyte-response pathways. Br J Dermatol 159: 1092-1102, 2008.

39. Chiricozzi A, Guttman-Yassky E, Suárez-Fariñas M, Nograles KE, Tian S, Cardinale I, Chimenti S and Krueger JG: Integrative responses to IL-17 and TNF- $\alpha$ in human keratinocytes account for key inflammatory pathogenic circuits in psoriasis. J Invest Dermatol 131: 677-687, 2011.

40. Rabeony H, Petit-Paris I, Garnier J, Barrault C, Pedretti N, Guilloteau K, Jegou JF, Guillet G, Huguier V, Lecron JC, et al: Inhibition of keratinocyte differentiation by the synergistic effect of IL-17A, IL-22, IL-1 $\alpha$, TNF $\alpha$ and oncostatin M. PLoS One 9: e101937, 2014.

41. Chandra A, Ray A, Senapati S and Chatterjee R: Genetic and epigenetic basis of psoriasis pathogenesis. Mol Immunol 64: 313-323, 2015.

42. Beaudoing E and Gautheret D: Identification of alternate polyadenylation sites and analysis of their tissue distribution using EST data. Genome Res 11: 1520-1526, 2001.

43. Boutrot F, Chantret N and Gautier MF: Genome-wide analysis of the rice and Arabidopsis non-specific lipid transfer protein (nsLtp) gene families and identification of wheat nsLtp genes by EST data mining. BMC Genomics 9: 86, 2008.

44. Skrabanek L and Campagne F: TissueInfo: High-throughput identification of tissue expression profiles and specificity. Nucleic Acids Res 29: E102, 2001

45. Mégy K, Audic S and Claverie JM: Heart-specific genes revealed by expressed sequence tag (EST) sampling. Genome Biol 3: RESEARCH0074, 2002.

46. Malone K, Sohocki MM, Sullivan LS and Daiger SP: Identifying and mapping novel retinal-expressed ESTs from humans. Mol Vis 5: 5, 1999. 
47. Wu TH, Chu LJ, Wang JC, Chen TW, Tien YJ, Lin WC and Ng WV: Meta-analytical biomarker search of EST expression data reveals three differentially expressed candidates. BMC Genomics 13 (Suppl 7): S12, 2012.

48. Gerber PA, Hevezi P, Buhren BA, Martinez C, Schrumpf H, Gasis M, Grether-Beck S, Krutmann J, Homey B and Zlotnik A: Systematic identification and characterization of novel human skin-associated genes encoding membrane and secreted proteins. PLoS One 8: e63949, 2013.

49. Chou RH, Wen HC, Liang WG, Lin SC, Yuan HW, Wu CW and Chang WS: Suppression of the invasion and migration of cancer cells by SERPINB family genes and their derived peptides. Oncol Rep 27: 238-245, 2012.

50. Hida T, Okura M, Kamiya T and Yamashita T: Nagashima-type palmoplantar keratosis caused by compound heterozygous mutations in SERPINB7. Eur J Dermatol 25: 202-203, 2015.

51. Shirakata Y, Kishimoto J, Tokumaru S, Yamasaki K, Hanakawa Y, Tohyama M, Sayama K and Hashimoto K: Epiregulin, a member of the EGF family, is over-expressed in psoriatic epidermis. J Dermatol Sci 45: 69-72, 2007.

52. Shirasawa S, Sugiyama S, Baba I, Inokuchi J, Sekine S, Ogino K, Kawamura Y, Dohi T, Fujimoto M and Sasazuki T: Dermatitis due to epiregulin deficiency and a critical role of epiregulin in immune-related responses of keratinocyte and macrophage. Proc Natl Acad Sci USA 101: 13921-13926, 2004.
53. Haneda T, Imai Y, Uchiyama R, Jitsukawa $\mathrm{O}$ and Yamanishi $\mathrm{K}$ : Activation of molecular signatures for antimicrobial and innate defense responses in skin with transglutaminase 1 deficiency. PLoS One 11: e0159673, 2016.

54. Dahlqvist J, Westermark GT, Vahlquist A and Dahl N: Ichthyin/NIPAL4 localizes to keratins and desmosomes in epidermis and Ichthyin mutations affect epidermal lipid metabolism. Arch Dermatol Res 304: 377-386, 2012.

55. Klar J, Schweiger M, Zimmerman R, Zechner R, Li H, Törmä H, Vahlquist A, Bouadjar B, Dahl N and Fischer J: Mutations in the fatty acid transport protein 4 gene cause the ichthyosis prematurity syndrome. Am J Hum Genet 85: 248-253, 2009.

56. Maier D, Mazereeuw-Hautier J, Tilinca M, Cosgarea R and Jonca N: Novel mutation in NIPAL4 in a Romanian family with autosomal recessive congenital ichthyosis. Clin Exp Dermatol 41: 279-282, 2016 International (CC BY-NC-ND 4.0) License. 